\title{
LA FORMACIÓN INICIAL DE PROFESORES DE HISTORIA Y CIENCIAS SOCIALES DESDE LA PERSPECTIVA DE LA DIDÁCTICA DE LA ESPECIALIDAD: FUNDAMENTOS, DESAFÍOS Y PROYECCIONES
}

\author{
Laura Valledor Cuevas \\ "In the future, as in the past, the fate of social studies \\ innovations will depend on educating the educators."
}

Stephen J. Thornton, 200I.

\begin{abstract}
RESUMEN
Este artículo aborda el rol de la Didáctica de la Historia y las Ciencias Sociales en la formación de profesores del área. Partiendo desde la evidencia disponible al respecto, generada por la tradición investigativa hispana y por la anglosajona, se afronta el análisis del impacto en la formación docente de las demandas sociales hacia el estudio escolar de la historia, así como también de las demandas provenientes desde la epistemología de las disciplinas sociales. Asimismo, se releva el problema de la tensión entre teoría y práctica en la formación de docentes y se expone una propuesta de trabajo para su superación.
\end{abstract}

Palabras clave: formación docente, enseñanza de la historia, conocimiento pedagógico del contenido.

\section{THE EARLY TRAINING OF TEACHERS OF HISTORY AND SOCIAL SCIENCE FROM THE PERSPECTIVE OF METHODOLOGY: FUNDAMENTALS, CHALLENGES AND PROJECTIONS}

\begin{abstract}
This article describes the role of History Didactics and Social Sciences in teacher education. The analysis of the impact in teacher education of the social requirements toward the school study of history is confronted, as well as the originating demands since the epistemologies of social disciplines from both the Hispanic and the Anglo-Saxon investigative traditions. Likewise, the problem of the tension between theory and practice in the teacher education is examined and finally, a proposal to approach this tension is exposed.
\end{abstract}

Keywords: teacher education, teaching history, pedagogical content knowledge.

* Candidata a doctora en Ciencias de la Educación, profesora de Didáctica de la Historia de la Facultad de Educación de la Pontificia Universidad Católica de Chile y directora institucional del proyecto de perfeccionamiento docente Talleres Comunales de Historia y Ciencias Sociales (desarrollado conjuntamente por CPEIP, PUC y DIBAM). E-mail: 1svalled@uc.cl, teléfono: 3545367. 


\section{Introducción}

A fines de la década de los ochenta, Beverly Armento realizó una síntesis de todos los estudios que se habían hecho en enseñanza y aprendizaje de las ciencias sociales. Uno de los problemas fundamentales que detectó, decía relación con la no inclusión de los hallazgos investigativos en un marco teórico proporcionado por las evidencias anteriores (Armento, I986 citado por Bravo, 2002:6I). Dos décadas más tarde, la situación de la Didáctica, esta vez en Chile, no parece diferente a la enunciada por Armento:

En Chile, aún no existen las didácticas específicas como disciplinas separadas de la pedagogía general. Los estudios son escasos y no tienen el rigor metodológico esperado para establecer un piso seguro de análisis. Las bibliografías y lecturas extranjeras no siempre se ajustaban del todo con los problemas y realidades nacionales, aunque en los últimos años se tuvieran puntos de encuentro mayores, por haber seguido modelos pedagógicos similares. (Vásquez, 2004: 24, 25)

A pesar de lo anterior, el momento actual nos parece un tanto más auspicioso. Desde hace algunos años, la formación de profesores tanto de Educación Básica como Media, incluye cursos de didáctica en prácticamente todas las universidades chilenas. En el ámbito específico de la historia y las ciencias sociales, se dispone de algunas instancias de especialización ${ }^{\mathrm{I}}$ y se encuentran en curso algunas tesis doctorales, que se sumarán a las ya finalizadas. Las dos tesis doctorales concluidas (Bravo, 2002 y Vásquez, 2004) se ocupan del tema que nos convoca aquí: la formación inicial de profesores de historia y ciencias sociales. Aunque solo la de Vásquez se refiere a la realidad chilena, ambas constituyen aportes importantes al tema.

Nos planteamos la formación inicial de profesores de Historia y Ciencias Sociales desde la perspectiva de la didáctica de la especialidad².

I La Universidad Alberto Hurtado fue pionera en la apertura de un diplomado en Didáctica de la Historia y Ciencias Sociales que se ha repetido con éxito desde el año 2003.

2 Utilizaremos los términos "didáctica de la historia" y "teaching bistory" como homónimos, a fin de 
Iniciaremos la reflexión describiendo la investigación disponible acerca de una de las sublíneas más importantes en lo que al estudio de la formación docente se refiere: las ideas previas de los estudiantes de pedagogía, asumiendo este tema como el punto de partida de cualquier programa exitoso de formación inicial de profesores. Continuaremos con una revisión a las demandas que la sociedad realiza al estudio de la historia y las ciencias sociales y que contribuyen a configurar el perfil del docente al que se espera arribar con los programas de formación inicial. En seguida, conduciremos la reflexión en torno a las características del saber disciplinar de los profesores de historia y ciencias sociales chilenos, para luego concluir abordando el problema de la tensión teoría-práctica de los programas de formación docente y la formulación de una propuesta para enfrentarlo de manera exitosa.

\section{Las ideas previas de los futuros profesores de Historia y Ciencias Sociales}

Al analizar la investigación disponible en la línea de investigación de la formación inicial de docentes, Banks y Parker (1992 citado por Bravo, 2002: 62) concluyeron que esta podía ser caracterizada por dos rasgos fundamentales: la existencia de visiones en conflicto acerca de lo que se considera una buena formación de profesores y la carencia de un cuerpo de investigación coherente en cuanto a la enseñanza y educación de los mismos. En adición a esta carencia, se encontraba la disconformidad, acusada por la literatura especializada, imperante en varios países en cuanto a la calidad de los programas de formación inicial de profesores de historia (Pagès, 1997: 52). Debido a lo anterior, resultaba urgente la conformación y desarrollo de un cuerpo de investigación acerca de la formación de profesores de ciencias sociales.

Actualmente, en el ámbito de la formación inicial del profesorado, pueden distinguirse tres sublíneas de investigación: las ideas previas

permitir un diálogo entre los aportes teóricos de los cuerpos investigativos de habla hispana y anglosajón. 
de los futuros profesores, el proceso de formación inicial y la relación teoría-práctica (Bravo, 2002: 57). En torno a la primera de las sublíneas mencionada, Joan Pagès puntualiza:

Las finalidades de esta línea de investigación consisten en indagar cuáles son las representaciones que tiene el estudiante de maestro sobre los contenidos que deberá enseñar, cuáles son sus orígenes y cuál es su racionalidad, y, en especial, qué estrategias deben seguirse en su formación para conseguir su cambio conceptual y evitar que dichas representaciones actúen como obstáculos para su capacitación didáctica. (Pagès, I999: I67)

Como ya se ha mencionado, el estudio de la formación inicial de docentes de historia y el de las ideas previas de los estudiantes de pedagogía, constituyen dos sublíneas investigativas distintas. Sin embargo, al revisar la investigación disponible resulta evidente la influencia que la última ejerce sobre la primera. Los trabajos al respecto señalan que las ideas que tienen los estudiantes que ingresan a estudiar pedagogía acerca de la historia en tanto disciplina y lo que la enseñanza de esta debe ser, inciden en el impacto que las actividades curriculares de un programa de formación pueda conseguir.

En este caso, las ideas previas están fuertemente arraigadas ya que los estudiantes de pedagogía las han aprendido lentamente y a través de su trayectoria académica como alumnos (Pagès, 1999: 167), es decir, en el transcurso de unos doce años de enseñanza escolar. A esta fuente de ideas previas, Pagès (Pagès 2000, citado por Bravo 2002) agrega las contribuciones efectuadas por los medios de comunicación o la familia.

El problema de las ideas previas de los estudiantes de pedagogía cobra su real dimensión al constatar el escaso impacto que tienen en estas, los cursos de didáctica de los programas de formación (Bravo, 2002: 70). Está claro que la presencia pertinaz de las ideas previas minimiza la influencia de los programas de formación docente, contribuyendo a la perpetuación de los rasgos tradicionales de la enseñanza de la historia y las ciencias sociales. Es por este motivo, en parte, que 
los avances producidos al interior de la disciplina historiográfica y también los construidos por la didáctica de la historia y las ciencias sociales en cuanto a estrategias de enseñanza y aprendizaje de conceptos y habilidades históricas, 'habitan en un domicilio' muy lejano de la residencia de las prácticas pedagógicas.

Benett y Spalding (1992 citado por Bravo, 2002: 7I) profundizan en esta problemática y plantean que las ideas previas de los profesores actúan como filtros ocultando información y seleccionando ideas que armonicen con pensamientos previos. Debido a esta demoledora realidad, la única forma de cambiar las percepciones de los profesores es la negociación y el diálogo en torno a estas. Del mismo modo, Benejam (200I citado por Bravo, 2002) pone el énfasis en la necesidad de comprender estas ideas previas para su posterior tratamiento en el proceso de formación de profesores.

En su tesis doctoral, Liliana Bravo pone de manifiesto que pese a todo el conocimiento recabado por la investigación didáctica, el estudio de las ideas previas de los estudiantes de pedagogía en historia y ciencias sociales, no siempre se ha traducido en propuestas para superar las mismas (Bravo, 2002: 69). La autora puntualiza la necesidad de emprender investigaciones que estudien las razones de la persistencia de las ideas previas después de completada la formación inicial docente. Pero, ante todo y como prioridad más acuciante, plantea la necesidad de conocer lo que los estudiantes de pedagogía piensan acerca de la enseñanza de la historia y el rol del profesor, en el momento mismo en que ingresan a un programa de formación docente, iniciando el proceso de educación desde estas:

La conducción de un proceso de enseñanza centrado en el tratamiento de modelos alternativos al modelo tradicional, sólo podrá concretarse en la medida en que el proceso de formación se desarrolle a partir de la reflexión de lo que se piensa de la enseñanza de las ciencias sociales y del papel del profesor, de manera que a través de esta reflexión permanente los futuros profesores cuestionen sus percepciones en función de optar por un modelo de enseñanza y un estilo docente que responda a una 
elección centrada en el conocimiento de alternativas educativas y no en la mera reproducción de lo conocido o protagonizado como alumnos (Bravo, 2002: 73)

El análisis de la sublínea de investigación de las ideas previas, permite establecer claramente cuál debe ser uno de los puntos de inicio de un programa de formación inicial de profesores de historia y ciencias sociales. Sin embargo, no existe la misma certeza al momento de definir hacia dónde debemos llegar. A nuestro juicio, esta falta de claridad podría radicar en la finalidad que cada sociedad otorga al estudio de la historia en el sistema escolar y que muchas veces posee distintas versiones al interior del mismo cuerpo social.

\section{Qué se espera de la formación inicial docente}

Tradicionalmente se ha asignado al estudio de la historia en la escuela el rol de formar ciudadanos (Rosa Rivero, 2004: 67). Sin embargo, la ciudadanía es un concepto que asume formas distintas dependiendo del lugar y el tiempo en que nos situemos. Por ejemplo, resulta sustancialmente distinto ser ciudadano en el Chile del siglo XIX, que en la actualidad. Mientras que durante el siglo XIX los ciudadanos eran un puñado de hombres notables, educados, mayores de edad y dotados de poder económico, hoy son también ciudadanos los desposeídos, comunes y corrientes, las mujeres, los analfabetos y los menores de edad, cuya imposibilidad de sufragar o ser electos en cargos públicos representa sólo una pequeña dimensión de la ciudadanía. ¿Cómo podríamos explicar la 'revolución pingüina' del año 2006 bajo el concepto de ciudadanía decimonónico?

El currículo nacional otorga a los subsectores de Estudio y Comprensión de la Sociedad e Historia y Ciencias Sociales -que bajo el Ajuste curricular se unifican en Historia, geografía y Ciencias Sociales- la finalidad de formar ciudadanos conscientes de su pertenencia a un grupo social dotado de una historia y cultura común y que sean miembros responsables y activos en una sociedad plural, solidaria y democrática (MINEDUC, 2005: 96-98; MINEDUC, 2002: I35). Ello implica, aunque se lea 
redundante, que quienes damos clase de didáctica de la historia en cualquier facultad de educación chilena, debemos 'formar formadores' de ciudadanos. En nuestra opinión, la complejidad de esta tarea no se aprecia del todo sino cuando observamos el contexto sociohistórico en el que esos futuros ciudadanos jugarán su rol. Un país económicamente pujante, donde los frutos de esa pujanza se distribuyen de un modo desigual y las diferencias de esta distribución pueden ser palpadas a nivel de habilidades y conocimientos de los alumnos, por cualquier profesor que haya ejercido en escuelas pertenecientes a más de una dependencia administrativa. Afirmamos esto, en primer lugar, porque la historia y las ciencias sociales están plagadas de conceptos abstractos y complejos, difíciles de enseñar a niños y adolescentes que muchas veces no cuentan con habilidades de base, tales como comprensión lectora o expresión oral. En segundo lugar, porque resulta altamente difícil convencer de participar a personas acostumbradas a estar al margen.

Pero, ¿qué características debe tener un buen profesor de historia y ciencias sociales para conducir el proceso de formación de ciudadanos que se le encomienda y, en definitiva, para ser un buen profesor de su área? Sin duda, la delimitación de este perfil encarna además el destino al que debe arribar cualquier programa serio de educación de profesores.

En Chile, el Marco Para la Buena Enseñanza (MBE) establece algunos parámetros acerca de lo que se considera un buen profesor. Sin embargo, dicha descripción está planteada en términos generales, es decir, sin distinción de especialidad disciplinar. Los programas de evaluación Docente Más y Acreditación de la Excelencia Pedagógica (AEP), se abocan a la medición del desempeño de docentes de historia y ciencias sociales -entre otras disciplinas- para lo cual toman como referencia el MBE. Mediante una prueba el programa AEP evalúa uno de los cuatro dominios de desempeño contemplados en el $\mathrm{MBE}^{3}$ :

3 Los dominios son: preparación para la enseñanza, creación de un ambiente propicio para el aprendizaje, enseñanza para el aprendizaje de todos los estudiantes y responsabilidades profesionales. 
Preparación de la enseñanza y dentro de este, solamente un criterio de ejercicio profesional: Domina los contenidos de las disciplinas que enseña y el marco curricular nacional (MINEDUC, 2003:II). Sin embargo, aunque AEP evalúa también otros dominios del MBE por medio de la construcción de un portafolio, esta evaluación carece de referencia específica a cada disciplina.

Hoy en día, podemos decir que ha habido un avance, desde la política pública, con el desarrollo del programa INICIA. Hasta el momento, este ha consistido en la evaluación formativa de los egresados de las carreras de pedagogía básica, y en el trabajo conjunto de distintas instituciones en la construcción de orientaciones curriculares y estándares para la formación docente. Una de las novedades de dicho programa ha sido la separación por especialidades en el caso de la prueba de conocimientos, con lo que se busca evaluar el conocimiento de la materia por parte de los egresados; pero, más interesante aún, ha sido el hecho de que para las orientaciones y estándares en desarrollo se hayan considerado lo disciplinario y lo pedagógico. De esta forma podríamos estar a las puertas, por primera vez, de plantearnos la formación docente a nivel nacional no solo como la adquisición de un dominio pedagógico general o un dominio disciplinar específico, sino como una compleja mixtura de ambas y además, de la necesidad de dominar el conocimiento pedagógico de cada disciplina.

El dominio de desempeño abordado por AEP representa una parte básica, pero incompleta de lo que la literatura especializada entiende por conocimiento pedagógico. En más de un autor es posible constatar que el conocimiento docente es un todo complejo que, al mismo tiempo que sobrepasa ampliamente el manejo de un área disciplinar, asume una forma distinta del conocimiento de los especialistas en esa área:

[El conocimiento pedagógico de los contenidos] representa la mezcla entre materia y pedagogía por la que se llega a una comprensión de cómo determinados temas y problemas se organizan, se representan y se adaptan a los diversos intereses y capacidades de los alumnos, y se exponen para su enseñanza. El conocimiento pedagógico de la materia es la categoría que con 
mayor probabilidad permite distinguir entre la comprensión del especialista en un área del saber y la comprensión del pedagogo. (Shulman, 1987: 175)

“... los profesores deben no sólo saber el contenido de la disciplina que enseñarán, sino también conocer métodos adecuados para transformarla para los propósitos de la instrucción. Esto hace al tratamiento sistemático del método una piedra angular de la formación de profesores" (Thornton, 200I: 73$)^{4}$

Lo que Thornton llama los métodos adecuados para transformar el conocimiento para la instrucción en el aula, sobrepasa las estrategias de enseñanza y abarca también los propósitos de la enseñanza escolar de la disciplina, los énfasis a efectuar y sus implicaciones sociales posteriores. Continuando con su razonamiento, el autor señala que los propósitos de la enseñanza de las ciencias sociales se hallan más allá de la disciplina misma, y de los métodos de enseñanza y que en última instancia, implican demostrar la relevancia del estudio de las ciencias sociales para el mundo en el cual los estudiantes viven en la actualidad. (Thornton, 2001:76)

Es debido a la diferencia existente entre conocimiento experto de un área y conocimiento pedagógico de la misma, que la formación de profesores debe asumir un carácter distinto a la formación de expertos disciplinares. El conocimiento pedagógico debe tener una doble base de sustentación: por una parte, el conocimiento disciplinar y por otra, el conocimiento de las formas de su enseñanza, aprendizaje y evaluación, además del conocimiento acabado acerca de los problemas de aprendizaje específicos que la disciplina implica para los estudiantes.

4 Las citas de bibliografía anglosajona que no está disponible en castellano se presentan en traducción libre realizada por la autora. 


\section{El dominio del conocimiento disciplinar en los docentes chilenos}

Hasta el momento, la única investigación empírica acerca del conocimiento disciplinar de profesores de historia chilenos, es la desarrollada por Nelson Vásquez. Vásquez (2004) estudió los estilos y las características principales de la formación universitaria inicial de los profesores de historia chilenos, así como también el perfeccionamiento permanente y la percepción por parte de los profesores de la utilidad de tales formaciones en el ejercicio profesional, llevando a cabo su estudio mediante un cuestionario aplicado a los profesores de la Quinta Región de Valparaíso. Algunas de las preguntas incluyeron los años de formación cursados, áreas de conocimiento estudiadas y las razones por las que eligieron la profesión docente. A nuestro entender, el ámbito de acción de este estudio queda definido por el dominio de las percepciones de los docentes, puesto que no nutre su trabajo ni de un análisis de las mallas curriculares de los programas de formación docente, ni de entrevistas a académicos universitarios que participaran de la formación inicial de profesores. Esta carencia en las fuentes de información nos lleva a matizar los hallazgos de su investigación en cuanto a su objetivo central: establecer los estilos y las características principales de la formación universitaria inicial de los profesores de historia.

Sin embargo, Vásquez otorga luces acerca de aspectos cruciales de lo que los docentes piensan de sí mismos, uno de estos se refiere a las fortalezas percibidas en su conocimiento profesional: "De las certezas que esos docentes tienen a la hora de enseñar, una es la seguridad en los conocimientos bistóricos aprendidos en la universidad." (Vásquez, 2004: 387)

En este punto cabe preguntarse ¿qué tipo de conocimientos históricos?, ¿bajo qué corriente historiográfica? Si la confianza de los profesores se basa en el dominio de datos históricos y cronología, no estamos en condiciones de afirmar que su formación disciplinar sea adecuada. El advenimiento de escuelas historiográficas tales como los Annales (por cierto, de larga data) y la Nueva Historia, así como las características de nuestra sociedad actual, en la que se dispone de manera automática 
de una gran cantidad de información, que además queda rápidamente caduca, plantean demandas que sobrepasan un conocimiento enciclopédico de la historia. Hablamos de la necesidad de contar con profesores que conozcan la epistemología de las ciencias que enseñan, sus metodologías y procesos de producción de conocimiento y más aún, que conozcan la especificidad en términos de razonamiento con la que estas ciencias pueden aportar a la formación intelectual de ciudadanos nuevos. En suma, hablamos de profesores que sepan, ante todo, qué es la historia, qué son las ciencias sociales y qué las diferencia de las ciencias exactas o biológicas. A nuestro juicio, el dominio de este tipo de conocimiento se encuentra la base de la toma de decisiones pedagógicas verdaderamente profesionales, puesto que permite dejar a la intuición en un segundo plano y fundamentar un curso de acción pedagógico en nociones genuinamente científicas. Vásquez prosigue en su relato de la formación inicial de profesores de historia:

Sin embargo, tales profesores de secundaria, aun cuando reconocen una formación marcada por la disciplina histórica, y en no pocas ocasiones indican que recibieron una instrucción para historiadores, también señalan en las respuestas que en dicha formación no se les proporcionó las herramientas básicas y adecuadas para construir conocimiento histórico. Como estudiantes universitarios tenían escaso acercamiento con archivos y fuentes documentales. Por sus manos nunca pasó un documento original y no saben qué hacer si se ven enfrentados a una fuente de primera mano.

Los profesores de secundaria no saben cómo construir hipótesis de investigación, cómo definir metodologías y cómo realizar un análisis histórico con fuentes. Por una formación de historiador, ellos suponen un dominio del relato histórico $y$ de los conocimientos factuales.

(Vásquez, 2004: 387, 388)

Las ausencias en la formación epistemológica de los profesores de historia aparecen delineadas de la manera más transparente, pero asumen un aspecto descarnado al contrastar el relato anterior con 
una de las definiciones de la profesión docente, presentes en la literatura experta:

La docencia es, esencialmente, una profesión ilustrada. Un profesor es miembro de una comunidad académica. Debe comprender las estructuras de la materia enseñada, los principios de la organización conceptual, como también los principios de indagación que ayudan a responder dos tipos de preguntas en cada ámbito: ¿cuáles son las ideas y las destrezas importantes en este campo?, y ¿de qué manera quienes generan conocimientos en esta área incorporan las nuevas ideas y descartan las deficientes? Vale decir, ¿cuáles son las reglas y los procedimientos de un saber académico y de la investigación de buen nivel? (Shulman, 1987: 176)

En nuestra opinión, el principal problema de las carencias evidenciadas en la formación de nuestros profesores de historia y ciencias sociales, no radica tanto en la falta de manejo de procedimientos historiográficos, sino en que la concepción expresada de lo que significa ser historiador, lleva implícita un concepto muy pobre de historia. Dicho de otro modo, si 'formarse como bistoriador' significa 'dominar el relato bistórico y los conocimientos factuales', resulta muy probable que ese relato y esos conocimientos sean la historia. Al despojar el conocimiento histórico de su metodología, se pasa por alto la incertidumbre presente en la interpretación de las fuentes y el carácter valórico e ideológico que atraviesa por completo la producción historiográfica (Appleby, Hunt y Jacob, I998: 225-253). En suma, se pasa por alto una de las realidades más evidentes de la disciplina: que la historia es un discurso construido por sujetos históricos que no pueden sustraerse a su carácter de tales y que por ende, sus valores, ideologías y, en suma, su época se encuentra presente en su relato aun cuando quieran evitarlo.

Esta realidad resulta virtualmente inaccesible si no se ha tenido la experiencia de trabajar con fuentes históricas. La concepción de la historia como un producto objetivo, acabado y aséptico está tan arraigada en los profesores y en la sociedad en general, que difícilmente 
puede ser cambiada mediante una 'declaración de principios' por parte de los formadores de profesores, ni aun por la más apasionada de las declaraciones. Se sabe a ciencia cierta que cada vez que enseñamos sin partir de las ideas previas de los aprendices, lo único que se consigue es la adquisición de conocimientos 'cosméticos' que no se incorporan a las estructuras cognitivas de un modo permanente, ni mucho menos sustentan las prácticas posteriores a su adquisición.

$\mathrm{Ni}$ siquiera las experiencias aisladas en el tratamiento de fuentes, rinden frutos en la formación de docentes de historia. En 2000 el investigador Samuel Wineburg dirigió una serie de talleres para profesores de ciencias sociales en Estados Unidos. El tema de los talleres era los libros de texto escolares y sus problemas en tanto recursos para aprender historia. En ellos se relevó como uno de los problemas más importantes de los textos de estudio la 'ilusión referencial' (Barthes citado por Wineburg, 1999: 8), es decir, el tipo de lenguaje que implica emitir un relato pasando por alto la referencia a otras fuentes y las posibles incertidumbres. Todos los participantes del taller reconocían el problema y sus implicancias pedagógicas, sin embargo, al planteárseles un problema histórico y entregarles las fuentes necesarias para resolverlo, el discurso histórico de los profesores tomó la misma forma de ilusión referencial de los libros de texto (Wineburg, 2000: 8). Experiencias como esta son la que dejan en evidencia la necesidad de un programa de formación de profesores de historia y ciencias sociales que contemple el tratamiento de fuentes como una práctica recursiva y constante.

Si bien lo que se enseña puede no ser lo mismo que lo que se aprende, un profesor que enseña una historia como producto objetivo y acabado, transmite el mensaje de que ya no resta nada por hacer. Keith Barton ha estudiado los formatos narrativos utilizados por los profesores para enseñar historia en Estados Unidos. Su principal hallazgo dice relación con la primacía de una narrativa de 'progreso nacional alimentada en el logro individual', con arreglo a la cual el país ha cursado etapas históricas sucesivas, cada una de las cuales ha dejado al país en una situación de bienestar mejor que la anterior y donde 
el cambio desde una etapa a otra se ha producido, no por el buen desempeño de instituciones o fuerzas sociales, sino por el empuje de individuos connotados. (Barton, 200I: 897)

Las implicancias sociales de este tipo de narrativa son cruciales. Si la historia de un país es presentada a través de una sola modalidad narrativa y mas aun, si esta modalidad implica un progreso lineal e indefinido, -que dicho sea de paso, se asemeja mucho al iluminismo decimonónico y constituye uno de los enfoques más añejos, gastados y superados de la historiografía- es muy sencillo sacar por conclusión que, sin importar lo que hagamos, las cosas siempre irán mejor:

Las limitaciones de esta clase de narrativas son, sin embargo, obvias. Esta falla en preparar a los niños para comprender la influencia de las instituciones sociales en la acción individual; falla en darles cuenta de la diversidad que existe en cualquier tiempo dado en la historia; falla en ilustrar el poder de la acción colectiva y falla en hacerles tomar conciencia de que la vida social y material no asciende invariablemente hacia niveles más cómodos y productivos de desarrollo. (Barton, 200I: 905)

A nuestro juicio, una formación de profesores que aborde las metodologías de producción historiográfica, además del dominio de procedimientos específicos, lleva aparejados dos aprendizajes fundamentales e indispensables. El primero de ellos es que un acontecimiento histórico se reconstruye desde la contrastación de varias fuentes primarias -y también secundarias- y que dentro de esta variedad es factible encontrar las versiones más disímiles y contradictorias frente al mismo hecho. El segundo aprendizaje es que las reconstrucciones del mismo hecho elaboradas por distintos historiadores, jamás son idénticas sino por el contrario, participan de una variedad muy similar a la de las fuentes primarias.

Es probable que el formato narrativo de los profesores de historia cuya formación contempló el tratamiento de fuentes históricas, difiera de aquellos quienes 'por sus manos nunca pasó un documento original y no saben qué bacer si se ven enfrentados a una fuente de primera mano' 
(Vásquez, 2004: 387, 388). No intentamos soportar por completo el peso de la formación docente en el conocimiento de la disciplina histórica y su epistemología. Una propuesta de esa índole, además de ilusa y anacrónica, se plantearía a contrapelo del cuerpo teórico existente y que ha caracterizado el conocimiento docente desde una multiplicidad de elementos que superan con mucho, el conocimiento disciplinar. Lo que sí sostenemos, es que la educación en competencias metodológicas disciplinares propias de la historiografía no puede estar ausente de un programa de formación de profesores de historia y ciencias sociales, sino que por el contrario, debe ser uno de los ejes de esta.

Ahora bien, en este punto del análisis la pregunta más asertiva parece ser ¿cómo logramos formar profesores con un dominio de la disciplina y del conocimiento pedagógico de esta? Al decir de Thornton, 'los profesores son "cuidadores del puente" entre curriculum $e$ instrucción. Pero, ¿cómo deberían ser formados para tender ese puente?' (Thornton, I99I. En Thornton, 2001: 72).

\section{Los programas de formación de profesores de historia y ciencias sociales}

Bravo afirma que al analizar un plan de formación de profesores ya existente o plantear uno nuevo, convergen múltiples factores: Contexto educativo y social en el que se desarrolla el programa, los currículos de los niveles de enseñanza para los que se está formando y los intereses de la sociedad (Bravo, 2002: 76). En estos tres elementos resultan fácilmente reconocibles temáticas pertenecientes a la didáctica de la historia y las ciencias sociales:

La didáctica de las Ciencias Sociales y la Historia se ocupa de estudiar las relaciones entre el profesor, el alumno y el saber escolar en el contexto de una clase y de un centro y en un momento histórico determinado, e investiga sus orígenes y tradiciones. Pretende elaborar conocimientos teórico-prácticos que permitan analizar y comprender qué ocurre cuando se 
enseña y se aprende Historia y Ciencias Sociales en contextos concretos y pensar alternativas para su enseñanza. (Pagès, 2004: I57)

Es evidente que la didáctica de la historia y las ciencias sociales tiene una responsabilidad enorme en la formación, tanto inicial como continua, de profesores. El corazón de la didáctica es su capacidad de articular teoría y práctica de un saber disciplinario en un contexto social e histórico particular. La coincidencia entre los elementos a considerar en el planteamiento y análisis de un plan de formación de profesores de historia y ciencias sociales y el objeto de estudio de la didáctica de estas disciplinas, hace que los conocimientos didácticos disponibles deban, necesariamente, informar el mejoramiento o creación de cualquier programa de formación docente.

Sin embargo, la realidad cuenta una historia distinta: a pesar de lo mucho que se ha avanzado, la didáctica de la historia y las ciencias sociales ha influido muy poco en la elaboración de programas de formación de profesores del área. Coincidimos con Beverly Armento (I996 citado por Bravo, 2002: 65), quien luego de un análisis exhaustivo del conocimiento acumulado hasta la fecha, relevó la necesidad imperiosa de dirigir el foco de la investigación al interior de los programas de formación de profesores. Creemos que es necesario emprender investigación que evalúe los programas de formación de profesores de historia y ciencias sociales disponibles en Chile. Es preciso poner en la palestra información 'dura' acerca de las carencias de la formación didáctica de profesores, capaz de fundamentar de un modo contundente las características de los modelos de formación que pudiéramos proponer y de este modo, hacer partícipe a la Didáctica en la toma de decisiones acerca de la formación de profesores.

Mientras tanto, la literatura especializada nos ofrece experiencia acerca de los elementos fundamentales a considerar en un plan de formación inicial docente. Un ejemplo de ello son los estudios de Pendry y Husbands, quienes exploraron las lecturas que los profesores de historia de educación media tuvieron durante su año 
de formación pedagógica y el valor que otorgaban a estas en el trabajo en aula posterior a su titulación (Pendry y Husbands, 2000). Los autores señalan que este estudio, aunque de manera vicaria y básica, exploró también la extensión en que los estudiantes veían su propio aprendizaje profesional como producto del conocimiento elaborado por la investigación. Las conclusiones fueron que aunque los profesores expresaban una valoración positiva de la producción científica de conocimiento pedagógico, en la práctica ese conocimiento competía con otras prioridades, de modo que los profesores recién egresados luchaban por otorgarle un espacio a un conocimiento que parecía no proveer de las respuestas para la actividad pedagógica cotidiana. Con evidente pesar, Pendry y Husbands señalan: "se da el caso, por supuesto, que los nuevos profesores pueden no ser la audiencia bacia la que se dirigen las investigaciones." (Pendry y Husbands, 2000: 332)

La realidad constatada por estos autores asume su real importancia si consideramos que los profesores de la población estudiada se formaron bajo la concepción de la profesión docente como una actividad basada en la investigación en la cual las prácticas son fundamentadas por los hallazgos de la investigación centrada, justamente, en la práctica:

Más recientemente, hemos notado que se ha visto desarrollar el concepto de enseñanza como una profesión basada en la investigación en la que el desarrollo del profesor es informado por los hallazgos de la investigación centrada en la práctica. Nuestra propia evidencia limitada sugiere que los fundamentos de una profesión basada en la investigación descansa en la formación inicial de profesores, ello es actualmente incierto. (Pendry y Husbands, 2000: 333)

En un ámbito 'vecino' al estudio recién señalado, se ha logrado establecer que las prácticas profesionales, los profesores tutores y los discursos que se plantean desde la esfera escolar y universitaria, ejercen una importante influencia en la formación inicial del profesorado. En torno a los centros de práctica y los profesores tutores, Booth llegó a la conclusión de que "el desarrollo y la capacitación como profesionales 
críticos y reflexivos sólo se puede lograr si los profesores de las escuelas asumen una actitud de colaboración con la formación de los futuros profesores" (Booth, I989 citado en Bravo, 2002: 78). Sin embargo, las facultades de educación y la escuela, son dos escenarios de formación que no necesariamente comparten intereses y objetivos (Pendry, 1990 citado en Bravo, 2002: 78) y cuya relación en el caso chileno adquiere la forma de un verdadero divorcio:

El eslabón perdido en el proceso chileno es una reforma de la formación de profesores en la cual la educación de los profesores se organice en relación estrecha con el currículo nacional y los profesores tengan mentores, "profesores maestros", que los acompañen de cerca en los inicios de su práctica y que se identifican por hacer una diferencia en el aprendizaje de los estudiantes. En el presente, el sistema de formación de profesores chileno está lejos de tener este grado de rigor y coherencia. Éste es claramente el desafío educacional más grande de Chile en el futuro próximo. (Carnoy, 2003: I22)

Al respecto sostenemos que es urgente propiciar la conjunción de los intereses de esos dos escenarios. A nuestro entender, la forma más sinérgica de lograrlo es que las facultades de educación asuman un rol de acompañamiento y asesoría a las unidades educativas del sistema escolar, mientras que estas últimas se involucren en la formación de profesores en y para la realidad de la educación chilena. Esta es una tarea que debe ser asumida por la didáctica de la historia y las ciencias sociales, debido a su capacidad de tender un puente entre la teoría y la práctica.

El 'reclutamiento' de profesores del sistema escolar para colaborar en la formación docente no puede ser un camino de una sola vía en el que los profesores tutores -también llamados colaboradores y mentores- sólo reciban a cambio el alivio temporal de sus funciones o la posibilidad de ausentarse del aula cuando el profesor en formación se encuentre atendiendo al curso. Una posibilidad concreta y factible es transformar la fase práctica de nuestros cursos de didáctica de la historia, en centros de elaboración de experiencias didácticas 
construidas sobre la base de la observación y detección de las características específicas del contexto de los alumnos de los profesores colaboradores. Además, sería justo asegurar la participación gratuita de los profesores 'mentores' en los programas de perfeccionamiento y magíster de las universidades con las que colaboran, lo que más que un esfuerzo por parte de las universidades, constituiría un beneficio puesto que lograría la alineación de los discursos facultad de educación profesores colaboradores, asegurando la coherencia del mensaje recibido por los profesores en formación. De este modo, la mantención de la calidad de profesor colaborador podría supeditarse a la participación en perfeccionamientos de la universidad, lo que en el ámbito municipalizado implica muchas veces un aumento en las remuneraciones de los profesores. Otro punto a considerar en el proceso de conjugación de intereses de estos dos escenarios, es el incentivo a los directores con asesorías educacionales gratuitas a sus escuelas, en ámbitos tales como la evaluación o el acompañamiento didáctico al aula.

Pero, en nuestra opinión, el rol de las facultades de educación en los centros escolares debería sobrepasar la asesoría y el acompañamiento. Es preciso que la universidad reconozca que los profesores en ejercicio construyen diariamente un conocimiento práctico riquísimo, pero del que rara vez son conscientes. Es urgente hacer de la escuela no sólo un centro de práctica para profesores novatos, sino un espacio de estudio de los profesores expertos y sus estrategias de enseñanza. Si así ocurriera, tal vez el trabajo de Pendry y Husbands citado más arriba relataría una situación distinta de la 'lucha' de los profesores recién titulados por incluir el conocimiento pedagógico de los libros en su práctica cotidiana. La producción de un conocimiento que, en algún grado, crece a contrapelo de la realidad nos parece un esfuerzo tan absurdo como salir de España hoy en día y tratar de llegar a las Indias navegando hacia el oeste. La colisión inminente con un continente ignoto y poblado de contradicciones, es una experiencia que no tenemos el derecho de obligar a vivir a nuestros estudiantes de pedagogía, no sin antes dotarlos de las armas para enfrentarla. En la medida en que exista una doble vía entre la escuela 
y la producción de conocimiento didáctico, será mucho más factible formar profesores de historia y ciencias sociales que respondan a las demandas múltiples nacidas desde la epistemología de las disciplinas, desde el curriculum, desde las fallas del sistema educacional o desde las inequidades socioculturales de los estudiantes.

\section{Bibliografía}

Appleby, J., Hunt, L y Jacob, M. (1998). La verdad sobre la bistoria. Editorial Andrés Bello, Santiago.

Barton, K. (200I). “A sociocultural perspective on children's understanding of historical change: Comparative findings from Northern Ireland and the United States." En: American Educational Research Journal. Volumen 38. $\mathrm{N}^{\circ}$ 4, Páginas 88I-9I3. American Educational Research Association, Washington D.C.

Bravo, L. (2002). La formación inicial del profesorado de secundaria en Didáctica de las Ciencias Sociales en la Universidad Autónoma de Barcelona: un estudio de caso. Tesis doctoral. Dirección: Joan Pagès. Departament de Didàctica de la Llengua, de la Literatura i de les Cièncis Socials. Universitat Autònoma de Barcelona.

Carnoy, M. (2003). "Las políticas educacionales de Chile desde una perspectiva internacional”. En Cox, C. (Ed.) Políticas educacionales en el cambio de siglo: la reforma del sistema escolar cbileno. Páginas II5-I22. Editorial Universitaria, Santiago.

MINEDUC (2002). Objetivos Fundamentales y Contenidos Mínimos Obligatorios de la Educación Básica. Santiago.

MINEDUC (2003). Marco para la Buena Enseñanza. Santiago.

MINEDUC (2005). Objetivos Fundamentales y Contenidos Mínimos Obligatorios de la Educación Media. Santiago.

Pagès, J. (1997). "La investigación sobre la formación inicial del profesorado para enseñar ciencias sociales". Asociación Universitaria del profesorado de Didáctica de las Ciencias Sociales: La formación del profesorado y la didáctica de las Ciencias Sociales. Páginas 49-86. Diada, Sevilla.

Pagès, J. (I999). "Las representaciones previas de los estudiantes de maestro de ciencias sociales, geografía e historia”. En: Teoría y Didáctica de las Ciencias Sociales. N ${ }^{\circ}$ 4, Páginas I6I-I78. Universidad de Los Andes, Facultad de Humanidades y Educación. Mérida, Venezuela. 
Pagès, J. (2004). "Enseñar a enseñar historia: La formación didáctica de los futuros profesores de historia” En: Nicolás, E. y Gómez, J. (coord.): Miradas a la Historia. Reflexiones bistoriográficas en recuerdo de Miguel Rodríguez Llopis (pp. I55-I78). Aula de debate. Universidad de Murcia.

Pendry, A.; Husbands, C. (2000). "Research and Practice in History Teacher Education” Cambridge Journal of Education. Volumen 30. Número 3, Páginas: 32I-334. Routledge Taylor \& Francis Group, Cambridge.

Rosa Rivero, A. (2004). "Memoria, historia e identidad. Una reflexión sobre el papel de la enseñanza de la historia en el desarrollo de la ciudadanía”. En: Carretero, M. y Voss, J. (Comp.) Aprender y pensar la bistoria (pp.: 47 a 69). Amorrortu Editores. Buenos Aires, Madrid.

Shulman, L. (1987). "Knowledge and Teaching. Foundations of the New Reform”, Harvard Educational Review. Volumen 57. Número I, primavera 1987. Traducción desde el inglés por Alberto Ide. Versión en castellano en Estudios Públicos, 83, invierno 200I. Páginas: 83 a 196.

Thornton, S. (200I). "Educating the Educators: Rethinking Subject Matter and Methods". Theory into Practice. Volumen 40, Número I, invierno, 2001. Páginas 72-78.

Vásquez, N. (2004). La formación del profesorado de bistoria en Chile. La formación inicial y permanente de los educadores de la $V$ Región en el marco de la reforma educacional. Tesis doctoral. Dirección: Joaquim Prats. Departamento de didáctica de las ciencias sociales. Universidad de Barcelona.

Wineburg, S. (1999). Historical thinking and other unnatural acts. Ganador del 1999 Exemplary Research in Social Studies Award of the Nacional Council for the Social Studies. Disponible en: http://www.pdkintl.org/kappan/ kwin9903.htm 